\title{
The in vivo growth and development of micropropagated Elatior begonias (Begonia $\times$ hiemalis). I. Study on the effect of lighting and substrate.
}

\author{
PÄIVI ROIVAINEN \\ Department of horticulture, University of Helsinki, \\ SF-00710 Helsinki, Finland
}

\begin{abstract}
Micropropagation is an efficient way to produce pathogen-free Elatior begonias. However, certain problems arise when these plants are used in commercial pot plant production. The plants grow too luxuriantly, the root collars remain thin and flowering is delayed.

To investigate the impact of the growing method, an experiment with different sources of supplementary light and different substrates was arranged in spring 1987. The following Elatior begonia cultivars were studied: 'Afrodite', 'Afrodite Special', 'Afrodite Rosa', 'Mandela', 'Barbara', 'Connie', 'Marja' and 'Sirene'. Four different lamp types were used: highpressure sodium lamps (Airam SNaKd $330 \mathrm{~W}$ ), high-pressure mercury lamps (Airam $\mathrm{HgLX}$ $400 \mathrm{~W}$ ), high-pressure metal halide lamps (Philips HPI/T $375 \mathrm{~W}$ ) and, as a control treatment, incandescent lamps of $100 \mathrm{~W}$. The plants were potted in B2-peat (Sphagnum peat with moderate fertilization and medium sieving grade), B2-peat ( 3 parts) plus perlite (1 part) or B2-peat ( 2 parts) plus perlite ( 2 parts).

The plants lighted with SNaKd or $\mathrm{HgLX}$ lamps were the highest, the broadest and the first to reach anthesis and the sale stage. The plants in the control group were the smallest and the last to reach anthesis and the sale stage. The effect of the substrate on the time required to reach anthesis and the sale stage was clear only in the cultivar 'Afrodite Special'; when potted in peat-perlite $2+2$, its plants flowered 2 weeks later than in other substrate mixtures. The height and the width of the plants were not greatly affected by the substrate. None of the lamp types or substrates clearly increased the number of flower buds or the diameter of the root collar. The proportion of saleable plants varied with the cultivar, lighting and substrate. In most cultivars it was below $50 \%$. The chief factors reducing saleability were luxuriant growth, weak flowering and oblique growth habit.
\end{abstract}

Index words: Begonia $\times$ hiemalis, micropropagation, lighting, substrate.

\section{Introduction}

Elatior begonia has become one of the most popular flowering pot plants in Finland. In 1986 almost 1,700,000 Elatior begonias were produced by 462 horticultural firms (ANON. 1987). Elatior begonias are traditionally propagated by stem or leaf cuttings, a method which, unfortunately, enables pathogens to be transferred from one generation of plants to 
another. One of the most serious diseases of Elatior begonia is bacterial leaf spot and blight caused by Xanthomonas begoniae. As no efficient chemical control of this disease is available at present, the only practical means of control is to prevent the spread of the disease. Micropropagation offers an efficient way to obtain healthy begonias at a high propagation rate.

The commercial growers have not, however, been completely satisfied with the quality of micropropagated Elatior begonias. According to them, the micropropagated plants grow too luxuriantly, the root collars remain thin and flowering is delayed compared with that of traditionally propagated plants. Due to the newness of this research field, very little information is available about the growth behaviour of micropropagated Elatior begonias. The flowering of these begonias has been studied by HiLding and Welander (1976) and Bigot (1981). They report that no significant variation was evident in flower shape, size or colour in micropropagated plants, compared with those propagated traditionally. Welander $(1978,1987)$ investigated the growth of clones from different micropropagated Elatior begonia cultivars and the impact of the season on the development of micropropagated Elatior begonias, but he does not mention having noticed unwanted growth habits.

The aim of this study was to investigate the growth and development of micropropagated Elatior begonias in supplementary lighting given by lamps with different spectral energy distributions and on substrate mixtures of different nutrient content and nutrient-holding capacity. Studies on supplementary lighting of traditionally propagated Elatior begonias have been published by HAGEN (1978) and MOE (1985).

\section{Materials and methods}

Eight cultivars of Elatior begonia were used in this experiment: 'Afrodite', 'Afrodite Special', 'Afrodite Rosa', 'Mandela', 'Barbara',
'Connie', 'Marja' and 'Sirene'. According to Hilding (1982), cultivars belonging to the 'Afrodite' group and 'Mandela' ('Ballerina' group) have vigorous growth, 'Barbara' and 'Connie' ("Daehnfeldt" group) grow moderately, and 'Sirene', belonging to the 'Nixe' group, shows restrained growth. No information was found in the literature about the growth vigour of 'Marja', but according to A. Pajunen (oral communication 24. 6. 1987), it belongs to the "Daehnfeldt" group.

The experimental plants had been micropropagated commercially and at the beginning of the experiment they were at the growth stage at which the commercial growers receive them, 4-10 cm high. Plants of every cultivar were divided into three groups according to the potting substrate to be used:

group 1: B2-peat (Sphagnum peat with $0.95 \%$ of $10-11.3-16$-fertilizer and $6.2 \%$ of dolomite lime by weight, medium sieving grade)

group 2: B2-peat (3 parts) + perlite (1 part) group 3: $\mathrm{B} 2$-peat (2 parts) + perlite ( 2 parts)

Perlite was used in order to obtain a lower nutrient content in the substrate just after potting. The plants were potted in $12 \mathrm{~cm}$ plastic pots and transferred to a greenhouse, where they were divided into four lighting groups, according to the lamp type to be used (different spectral energy distributions):

group 1: Airam SNaKd $330 \mathrm{~W}$ (high-pressure sodium lamps)

group 2: Airam $\mathrm{HgLX} 400 \mathrm{~W}$ (high-pressure mercury lamps)

group 3: Philips HPI/T $375 \mathrm{~W}$ (high-pressure metal halide lamps)

group 4: control (incandescent lamps $100 \mathrm{~W}$ )

In lighting groups $1-3$ the amount of supplementary radiant energy was $5000 \mathrm{~mW} /$ $\mathrm{m}^{2}( \pm 10 \%)$, i.e. $1700-2200$ lux at the level of the plant, depending on the lamp type. Group 4 was a control group, receiving supplementary light of 150-200 lux at plant level in order to keep the plants vegetative. The lighting was started immediately after potting 
on 16. 1. 1987 and was continued for 4 weeks, until 15. 2. 1987. The daily light-dark cycle was 16 hours light, 8 hours dark.

After the lighting period, the plants received a short-day treatment of 2 weeks, during which the light-dark cycle was 8 hours light, 16 hours dark. There was no supplementary lighting. After the short-day treatment the plants were given light with incandescent lamps (150-200 lux) at $04.00-08.00$ hours and at $16.00-20.00$ hours, in order to provide long-day conditions (16 hours), which promote formation and development of flower buds (HiLding 1982). Lighting was continued until 1. 4.1987, after which the natural daylength was sufficient.

The night temperatures during the different growing stages were intended to be as follows: vegetative period $+18^{\circ} \mathrm{C}$, short-day treatment $+20^{\circ} \mathrm{C}$ and time to flowering $+18^{\circ} \mathrm{C}$. In practice, however, these temperatures were difficult to keep and the night temperature range observed was $+11-+18^{\circ} \mathrm{C}$ (veg.), $+13-+20^{\circ} \mathrm{C}$ (short-day) and $+13-+18^{\circ} \mathrm{C}$ (flow.). Fertilizing was begun 3 weeks after potting. A fertilizer with $14 \% \mathrm{~N}, 5 \% \mathrm{P}$, $21 \% \mathrm{~K}$ plus micronutrients was used. Nutrients were applied at every watering at the rate of $70 \mathrm{ppm} \mathrm{N}, 24 \mathrm{ppm} \mathrm{P}$ and $105 \mathrm{ppm} \mathrm{K}$ for one week and thereafter at the rate of $112 \mathrm{ppm}$ $\mathrm{N}, 38 \mathrm{ppm} \mathrm{P}$ and $168 \mathrm{ppm} \mathrm{K}$ throughout the experiment. No growth regulators were used.

A randomized complete block design was used in this experiment with 6 replications. Observations were made at the beginning, at the end of the lighting treatment and from the beginning of anthesis to the end of the experiment. Height was measured from the pot rim to the highest peak of the plant. Width was determined as the average of two measurements at right angles to each other. The beginning of anthesis was determined as the date when the first open flower was noted. The sale stage was determined as the date when 7 flowers ('Afrodite' group and 'Mandela'), 6 flowers ('Barbara', 'Connie' and 'Marja') or 5 flowers ('Sirene') were open. The diameter of the root collar was measured on the top of the substrate from the firstflowering branch.

The height and the width of the individual plants were recorded immediately after potting and at the end of lighting. When a plant had reached the sale stage, the experiment was terminated for its part and the heigth, width, diameter of the root collar and saleability were observed. As there are no official requirements concerning the saleability of Elatior begonias, a subjective judgement was made and thus the results in Table 10 are merely indicative. The whole experiment was terminated on 29. 5. 1987, i.e. 19 weeks after potting and 15 weeks after the beginning of the short-day treatment. An analysis of variance was made and the means were separated by the use of an MSD, $5 \%$ level.

\section{Results}

\subsection{After lighting}

The results obtained after lighting are presented in Tables 1 and 2. In most cultivars, plants lighted with $\mathrm{SNaKd}$ lamps and plants potted in peat showed the greatest increase in height and width. $\mathrm{HgLX}$ lamps generally induced more growth than HPI/T lamps, but the differences were not significant, except in the increase of width of the cultivar 'Afrodite'. In most cases, plants grown under incandescent lamps and plants potted in peatperlite $2+2$ had the poorest growth, though the plants under incandescent lamps were not the lowest.

\subsection{At the sale stage}

Plants grown under $\mathrm{SNaKd}$ or $\mathrm{HgLX}$ lamps, depending on the cultivar, were the first to reach anthesis and the sale stage (Tables 3 and 4). The longest time till anthesis and the sale stage was observed under incandescent lamps. The differences between the lighting groups in reaching anthesis were $3-10$ days and in reaching the sale stage 6-11 days, depending on the cultivar. Peat- 
Table 1. Effect of lighting and substrate on the increase of height $(\mathrm{cm})$ in Elatior begonias (4 weeks after potting).

\begin{tabular}{|c|c|c|c|c|c|c|c|c|c|}
\hline \multirow[b]{2}{*}{ Treatment } & \multicolumn{8}{|c|}{ Cultivar } & \multirow[b]{2}{*}{$\begin{array}{c}\text { Treatment } \\
\text { mean }\end{array}$} \\
\hline & 'Afrodite' & $\begin{array}{l}\text { 'Afrodite } \\
\text { Special' }\end{array}$ & $\begin{array}{l}\text { 'Afrodite } \\
\text { Rosa' }\end{array}$ & 'Mandela' & 'Barbara' & 'Connie' & 'Marja' & 'Sirene' & \\
\hline \multicolumn{10}{|l|}{ Lighting: } \\
\hline SNaKd $330 \mathrm{~W}$ & $4.8 \mathrm{a}$ & $4.9 \mathrm{a}$ & $3.9 \mathrm{a}$ & $3.6 \mathrm{a}$ & $2.0 \mathrm{a}$ & $3.8 \mathrm{a}$ & $3.0 \mathrm{a}$ & $2.2 \mathrm{a}$ & 3.5 \\
\hline $\mathrm{HgLX} 400 \mathrm{~W}$ & $4.0 \mathrm{a}$ & $4.0 \mathrm{a}$ & $2.9 \mathrm{a}$ & $3.3 \mathrm{a}$ & $2.6 \mathrm{a}$ & $3.6 \mathrm{ab}$ & $3.0 \mathrm{a}$ & $2.1 \mathrm{a}$ & 3.2 \\
\hline $\mathrm{HPI} / \mathrm{T} 375 \mathrm{~W}$ & $3.7 \mathrm{a}$ & $3.8 \mathrm{a}$ & $3.0 \mathrm{a}$ & $2.8 \mathrm{a}$ & $2.1 \mathrm{a}$ & $3.0 \mathrm{ab}$ & $2.6 \mathrm{a}$ & $2.4 a$ & 3.0 \\
\hline incandescent $100 \mathrm{~W}$ & $4.2 \mathrm{a}$ & $3.9 \mathrm{a}$ & $3.8 \mathrm{a}$ & $3.5 \mathrm{a}$ & $2.3 \mathrm{a}$ & $2.9 \mathrm{~b}$ & $3.0 \mathrm{a}$ & $2.7 \mathrm{a}$ & 3.3 \\
\hline \multicolumn{10}{|l|}{ Substrate: } \\
\hline peat & $4.1 \mathrm{a}$ & $4.0 \mathrm{a}$ & $3.9 \mathrm{a}$ & $3.8 \mathrm{a}$ & $2.6 \mathrm{a}$ & $3.5 \mathrm{a}$ & $3.1 \mathrm{a}$ & $2.1 \mathrm{a}$ & 3.4 \\
\hline peat-perlite $3+1$ & $4.4 \mathrm{a}$ & $4.9 b$ & $3.3 \mathrm{a}$ & $3.7 \mathrm{a}$ & $2.4 \mathrm{a}$ & $3.4 \mathrm{a}$ & $3.0 \mathrm{a}$ & $2.8 \mathrm{a}$ & 3.5 \\
\hline peat-perlite $2+2$ & $4.0 \mathrm{a}$ & $3.6 \mathrm{a}$ & $3.0 \mathrm{a}$ & $2.5 b$ & $1.9 \mathrm{a}$ & $3.0 \mathrm{a}$ & $2.6 \mathrm{a}$ & $2.2 \mathrm{a}$ & 2.8 \\
\hline Cultivar mean & 4.2 & 4.2 & 3.4 & 3.3 & 2.3 & 3.3 & 2.9 & 2.4 & \\
\hline
\end{tabular}

Means marked with the same letter in one column do not differ at the $5 \%$ rate of error.

Table 2. Effect of lighting and substrate on the increase of width $(\mathrm{cm})$ in Elatior begonias (4 weeks after potting).

\begin{tabular}{|c|c|c|c|c|c|c|c|c|c|}
\hline \multirow[b]{2}{*}{ Treatment } & \multicolumn{8}{|c|}{ Cultivar } & \multirow[b]{2}{*}{$\begin{array}{c}\text { Treatment } \\
\text { mean }\end{array}$} \\
\hline & 'Afrodite' & $\begin{array}{l}\text { 'Afrodite } \\
\text { Special' }\end{array}$ & $\begin{array}{l}\text { 'Afrodite } \\
\text { Rosa' }\end{array}$ & 'Mandela' & 'Barbara' & 'Connie' & 'Marja' & 'Sirene' & \\
\hline \multicolumn{10}{|l|}{ Lighting: } \\
\hline SNaKd $330 \mathrm{~W}$ & $7.7 \mathrm{a}$ & $8.3 \mathrm{a}$ & $5.8 \mathrm{a}$ & $4.2 \mathrm{a}$ & $5.0 \mathrm{a}$ & $5.7 \mathrm{a}$ & $5.2 \mathrm{a}$ & $3.5 \mathrm{a}$ & 5.7 \\
\hline $\mathrm{HgLX} 400 \mathrm{~W}$ & $7.8 \mathrm{a}$ & 7.6ab & $5.9 \mathrm{a}$ & $4.1 \mathrm{a}$ & $4.0 \mathrm{a}$ & $5.2 \mathrm{ab}$ & $5.2 \mathrm{a}$ & $3.3 \mathrm{a}$ & 5.4 \\
\hline $\mathrm{HPI} / \mathrm{T} 375 \mathrm{~W}$ & $6.4 \mathrm{~b}$ & $6.2 \mathrm{~b}$ & $6.0 \mathrm{a}$ & $3.3 \mathrm{a}$ & $4.5 \mathrm{a}$ & $4.9 \mathrm{ab}$ & $4.2 \mathrm{ab}$ & $3.1 \mathrm{a}$ & 4.8 \\
\hline incandescent $100 \mathrm{~W}$ & $5.8 \mathrm{~b}$ & $6.0 \mathrm{~b}$ & $5.3 \mathrm{a}$ & $3.4 \mathrm{a}$ & $4.2 \mathrm{a}$ & $4.4 \mathrm{~b}$ & $4.0 \mathrm{~b}$ & $3.0 \mathrm{a}$ & 4.5 \\
\hline \multicolumn{10}{|l|}{ Substrate: } \\
\hline peat & $8.2 \mathrm{a}$ & $7.7 \mathrm{a}$ & $6.3 \mathrm{a}$ & $4.2 \mathrm{a}$ & $5.2 \mathrm{a}$ & $5.5 \mathrm{a}$ & $4.8 \mathrm{a}$ & $3.4 \mathrm{a}$ & 5.6 \\
\hline peat-perlite $3+1$ & $7.3 \mathrm{a}$ & $7.3 \mathrm{a}$ & $6.0 \mathrm{ab}$ & $3.8 \mathrm{a}$ & $4.4 \mathrm{ab}$ & $5.3 \mathrm{ab}$ & $4.9 \mathrm{a}$ & $3.3 \mathrm{a}$ & 5.3 \\
\hline peat-perlite $2+2$ & $5.4 \mathrm{~b}$ & $6.1 \mathrm{~b}$ & $5.0 \mathrm{~b}$ & $3.3 \mathrm{a}$ & $3.9 \mathrm{~b}$ & $4.4 \mathrm{~b}$ & $4.4 \mathrm{a}$ & $3.1 \mathrm{a}$ & 4.4 \\
\hline Cultivar mean & 6.9 & 7.0 & 5.8 & 3.7 & 4.5 & 5.1 & 4.7 & 3.3 & \\
\hline
\end{tabular}

Means marked with the same letter in one column do not differ at the $5 \%$ rate of error.

Table 3. Effect of lighting and substrate on the beginning of anthesis (days from the beginning of short-day treatment) in Elatior begonias.

\begin{tabular}{|c|c|c|c|c|c|c|c|c|c|}
\hline \multirow[b]{2}{*}{ Treatment } & \multicolumn{8}{|c|}{ Cultivar } & \multirow[b]{2}{*}{$\begin{array}{c}\text { Treatmen } \\
\text { mean }\end{array}$} \\
\hline & 'Afrodite' & $\begin{array}{l}\text { 'Afrodite } \\
\text { Special' }\end{array}$ & $\begin{array}{l}\text { 'Afrodite } \\
\text { Rosa' }\end{array}$ & 'Mandela' & 'Barbara' & 'Connie' & 'Marja' & 'Sirene' & \\
\hline \multicolumn{10}{|l|}{ Lighting: } \\
\hline SNaKd $330 \mathrm{~W}$ & $37 \mathrm{ab}$ & $56 a$ & $46 a$ & $54 \mathrm{a}$ & $62 \mathrm{a}$ & $38 \mathrm{a}$ & $50 \mathrm{a}$ & $48 \mathrm{a}$ & 49 \\
\hline $\mathrm{HgLX} 400 \mathrm{~W}$ & $33 \mathrm{a}$ & $61 \mathrm{a}$ & $43 \mathrm{a}$ & $55 \mathrm{a}$ & $66 \mathrm{a}$ & $39 a$ & $46 \mathrm{a}$ & $54 \mathrm{a}$ & 50 \\
\hline $\mathrm{HPI} / \mathrm{T} 375 \mathrm{~W}$ & $37 a b$ & $61 \mathrm{a}$ & $45 \mathrm{a}$ & $58 \mathrm{~b}$ & $65 a$ & $40 \mathrm{a}$ & $52 \mathrm{ab}$ & $50 \mathrm{a}$ & 51 \\
\hline incandescent $100 \mathrm{~W}$ & $42 b$ & $61 \mathrm{a}$ & $46 a$ & $59 b$ & $68 \mathrm{a}$ & $48 b$ & $60 \mathrm{~b}$ & $54 \mathrm{a}$ & 55 \\
\hline \multicolumn{10}{|l|}{ Substrate: } \\
\hline peat & $37 \mathrm{a}$ & $55 \mathrm{a}$ & $45 \mathrm{a}$ & $56 a$ & $64 a$ & $42 \mathrm{a}$ & $52 \mathrm{a}$ & $54 \mathrm{a}$ & 51 \\
\hline peat-perlite $3+1$ & $36 a$ & $54 a$ & $44 a$ & $56 a$ & $64 a$ & $41 \mathrm{a}$ & $48 \mathrm{a}$ & $50 \mathrm{a}$ & 49 \\
\hline peat-perlite $2+2$ & $39 a$ & $70 \mathrm{~b}$ & $46 a$ & $58 \mathrm{a}$ & $67 a$ & $41 \mathrm{a}$ & $55 \mathrm{a}$ & $50 \mathrm{a}$ & 53 \\
\hline Cultivar mean & 37 & 60 & 45 & 57 & 65 & 41 & 52 & 51 & \\
\hline
\end{tabular}

Means marked with the same letter in one column do not differ at the $5 \%$ rate of error. 
Table 4. Effect of lighting and substrate on time taken to reach the sale stage (days from the beginning of shortday treatment) in Elatior begonias.

\begin{tabular}{|c|c|c|c|c|c|c|c|c|c|}
\hline \multirow[b]{2}{*}{ Treatment } & \multicolumn{8}{|c|}{ Cultivar } & \multirow[b]{2}{*}{$\begin{array}{c}\text { Treatment } \\
\text { mean }\end{array}$} \\
\hline & 'Afrodite' & $\begin{array}{l}\text { 'Afrodite } \\
\text { Special' }\end{array}$ & $\begin{array}{l}\text { 'Afrodite } \\
\text { Rosa' }\end{array}$ & 'Mandela' & 'Barbara' & 'Connie' & 'Marja' & 'Sirene' & \\
\hline \multicolumn{10}{|l|}{ Lighting: } \\
\hline SNaKd 330 W & $58 \mathrm{ab}$ & $77 a$ & $68 \mathrm{a}$ & $66 a$ & $77 \mathrm{a}$ & $56 a$ & $69 \mathrm{ab}$ & $60 \mathrm{a}$ & 66 \\
\hline $\mathrm{HgLX} 400 \mathrm{~W}$ & $54 \mathrm{a}$ & $79 a$ & $66 a$ & $66 a$ & $80 \mathrm{a}$ & $57 a$ & $65 a$ & $65 a$ & 66 \\
\hline $\mathrm{HPI} / \mathrm{T} 375 \mathrm{~W}$ & $57 \mathrm{ab}$ & $83 a$ & $67 a$ & $69 a$ & $79 a$ & $60 \mathrm{a}$ & $68 \mathrm{ab}$ & $63 a$ & 68 \\
\hline incandescent $100 \mathrm{~W}$ & $63 b$ & $86 a$ & $72 \mathrm{a}$ & $74 b$ & $84 a$ & $68 b$ & $76 b$ & $67 a$ & 74 \\
\hline \multicolumn{10}{|l|}{ Substrate: } \\
\hline peat & $57 \mathrm{a}$ & $78 \mathrm{a}$ & $68 \mathrm{a}$ & $67 a$ & $76 a$ & $59 \mathrm{a}$ & $69 a$ & $66 a$ & 68 \\
\hline peat-perlite $3+1$ & $56 a$ & $78 \mathrm{a}$ & $66 a$ & $66 a$ & $80 \mathrm{a}$ & $60 \mathrm{a}$ & $67 a$ & $63 a$ & 67 \\
\hline peat-perlite $2+2$ & $61 \mathrm{a}$ & $89 b$ & $70 \mathrm{a}$ & $73 b$ & $83 a$ & $61 \mathrm{a}$ & $73 \mathrm{a}$ & $64 a$ & 72 \\
\hline Cultivar mean & 58 & 82 & 68 & 69 & 80 & 60 & 70 & 64 & \\
\hline
\end{tabular}

Means marked with the same letter in one column do not differ at the $5 \%$ rate of error.

Table 5. Effect of lighting and substrate on the number of flowers and flower buds in Elatior begonias.

\begin{tabular}{|c|c|c|c|c|c|c|c|c|c|}
\hline \multirow[b]{2}{*}{ Treatment } & \multicolumn{8}{|c|}{ Cultivar } & \multirow[b]{2}{*}{$\begin{array}{c}\text { Treatment } \\
\text { mean }\end{array}$} \\
\hline & 'Afrodite' & $\begin{array}{l}\text { 'Afrodite } \\
\text { Special' }\end{array}$ & $\begin{array}{l}\text { 'Afrodite } \\
\text { Rosa' }\end{array}$ & 'Mandela' & 'Barbara' & 'Connie' & 'Marja' & 'Sirene' & \\
\hline \multicolumn{10}{|l|}{ Lighting: } \\
\hline SNaKd $330 \mathrm{~W}$ & $45 a$ & $35 \mathrm{a}$ & $40 \mathrm{a}$ & $31 \mathrm{a}$ & $55 \mathrm{a}$ & $21 \mathrm{a}$ & $19 a$ & $25 \mathrm{a}$ & 34 \\
\hline $\mathrm{HgLX} 400 \mathrm{~W}$ & $33 \mathrm{a}$ & $31 \mathrm{a}$ & $30 \mathrm{a}$ & $32 \mathrm{a}$ & $56 a$ & $20 \mathrm{a}$ & $22 \mathrm{a}$ & $32 \mathrm{a}$ & 32 \\
\hline $\mathrm{HPI} / \mathrm{T} 375 \mathrm{~W}$ & $48 \mathrm{a}$ & $36 a$ & $31 \mathrm{a}$ & $31 \mathrm{a}$ & $50 \mathrm{a}$ & $22 \mathrm{a}$ & $20 \mathrm{a}$ & $24 \mathrm{a}$ & 33 \\
\hline incandescent $100 \mathrm{~W}$ & $39 a$ & $29 a$ & $30 \mathrm{a}$ & $26 a$ & $48 \mathrm{a}$ & $24 \mathrm{a}$ & $22 \mathrm{a}$ & $24 \mathrm{a}$ & 30 \\
\hline \multicolumn{10}{|l|}{ Substrate: } \\
\hline peat & $42 \mathrm{a}$ & $33 a$ & $33 \mathrm{a}$ & $31 \mathrm{ab}$ & $53 a$ & $22 \mathrm{ab}$ & $22 \mathrm{a}$ & $28 \mathrm{a}$ & 33 \\
\hline peat-perlite $3+1$ & $36 a$ & $33 a$ & $33 a$ & $32 \mathrm{a}$ & $53 \mathrm{a}$ & $24 \mathrm{a}$ & $22 \mathrm{a}$ & $26 \mathrm{a}$ & 33 \\
\hline peat-perlite $2+2$ & $44 a$ & $32 \mathrm{a}$ & $33 \mathrm{a}$ & $26 b$ & $51 \mathrm{a}$ & $19 b$ & $18 \mathrm{~b}$ & $24 \mathrm{a}$ & 31 \\
\hline Cultivar mean & 41 & 33 & 33 & 30 & 52 & 22 & 21 & 26 & \\
\hline
\end{tabular}

Means marked with the same letter in one column do not differ at the $5 \%$ rate of error.

perlite $3+1$ proved to slightly accelerate anthesis and the sale stage. Peat-perlite $2+2$ had the opposite effect in most cases. The differences between the substrate groups in reaching anthesis were $1-16$ days and in reaching the sale stage $2-11$ days, depending on the cultivar. In most cultivars, however, the differences were not significant. In the cultivar 'Afrodite Special' potting in peat-perlite $2+2$ strongly retarded anthesis and the sale stage.

Fifteen per cent of the plants of 'Afrodite Special', $4 \%$ of 'Afrodite Rosa' and $6 \%$ of 'Marja' had not reached the sale stage by the end of the experiment. The increase of height and width and the number of flowers and flower buds in these groups were as follows:

\begin{tabular}{lccc}
\hline Cultivar & $\begin{array}{c}\text { Increase } \\
\text { of height } \\
(\mathrm{cm})\end{array}$ & $\begin{array}{c}\text { Increase } \\
\text { of width } \\
(\mathrm{cm})\end{array}$ & $\begin{array}{c}\text { No. of } \\
\text { flowers and } \\
\text { flower buds }\end{array}$ \\
\hline 'Afrodite Special' & 15.8 & 31.0 & 10 \\
'Afrodite Rosa' & 11.7 & 24.8 & 14 \\
'Marja' & 11.1 & 16.5 & 12 \\
\hline
\end{tabular}

The number of flowers and flower buds (Table 5) was not clearly dependent on the different treatments.

The measurements made at the sale stage 
Table 6. Effect of lighting and substrate on the final increase of height $(\mathrm{cm})$ in Elatior begonias.

\begin{tabular}{|c|c|c|c|c|c|c|c|c|}
\hline \multirow[b]{2}{*}{ Treatment } & \multicolumn{7}{|c|}{ Cultivar } & \multirow[b]{2}{*}{$\begin{array}{c}\text { Treatment } \\
\text { mean }\end{array}$} \\
\hline & $\begin{array}{l}\text { 'Afrodite } \\
\text { Special' }\end{array}$ & $\begin{array}{l}\text { 'Afrodite } \\
\text { Rosa' }\end{array}$ & 'Mandela' & 'Barbara' & 'Connie' & 'Marja' & 'Sirene' & \\
\hline \multicolumn{9}{|l|}{ Lighting: } \\
\hline SNaKd $330 \mathrm{~W}$ & $16.2 \mathrm{a}$ & $14.2 \mathrm{a}$ & $17.2 \mathrm{a}$ & $13.2 \mathrm{a}$ & $12.6 \mathrm{a}$ & $11.4 \mathrm{a}$ & $8.8 \mathrm{a}$ & 13.8 \\
\hline $\mathrm{HgLX} 400 \mathrm{~W}$ & $13.3 \mathrm{~b}$ & $12.6 \mathrm{ab}$ & $16.0 \mathrm{ab}$ & $12.7 \mathrm{a}$ & $11.9 \mathrm{a}$ & $10.6 \mathrm{a}$ & $8.9 \mathrm{a}$ & 12.6 \\
\hline $\mathrm{HPI} / \mathrm{T} 375 \mathrm{~W}$ & $15.2 \mathrm{ab}$ & $14.0 \mathrm{a}$ & $14.5 b$ & $11.3 \mathrm{a}$ & $11.4 \mathrm{ab}$ & $10.9 \mathrm{a}$ & $8.6 \mathrm{a}$ & 12.6 \\
\hline incandescent $100 \mathrm{~W}$ & $12.4 \mathrm{~b}$ & $11.3 \mathrm{~b}$ & $11.9 \mathrm{c}$ & $12.2 \mathrm{a}$ & $10.2 \mathrm{~b}$ & $10.2 \mathrm{a}$ & $7.2 \mathrm{~b}$ & 10.9 \\
\hline \multicolumn{9}{|c|}{ Substrate: } \\
\hline peat & $13.4 \mathrm{a}$ & $12.9 \mathrm{a}$ & $16.2 \mathrm{a}$ & $12.1 \mathrm{a}$ & $12.0 \mathrm{a}$ & $10.0 \mathrm{a}$ & $8.6 \mathrm{a}$ & 12.5 \\
\hline peat-perlite $3+1$ & $15.1 \mathrm{a}$ & $13.3 \mathrm{a}$ & $15.3 \mathrm{a}$ & $12.6 \mathrm{a}$ & $11.8 \mathrm{ab}$ & $11.2 \mathrm{a}$ & $8.4 \mathrm{a}$ & 12.8 \\
\hline peat-perlite $2+2$ & $14.3 \mathrm{a}$ & $12.8 \mathrm{a}$ & $13.2 \mathrm{~b}$ & $12.5 \mathrm{a}$ & $10.9 \mathrm{~b}$ & $11.2 \mathrm{a}$ & $8.1 \mathrm{a}$ & 12.1 \\
\hline Cultivar mean & 14.3 & 13.0 & 14.9 & 12.4 & 11.6 & 10.8 & 8.4 & \\
\hline
\end{tabular}

Means marked with the same letter in one column do not differ at the $5 \%$ rate of error.

showed that the height and width of the plants were increased most by SNaKd lamps and, in some cultivars, by $\mathrm{HgLX}$ lamps. Plants grown under incandescent lamps were the lowest and the narrowest. The differences between the substrate groups were not very pronounced and depended on the cultivar (Tables 6 and 8). A very significant interaction $(F=5.87$ $+++)$ between lighting and substrate was

Table 7. Effect of lighting and substrate on the final increase of height in the Elatior begonia 'Afrodite'.

\begin{tabular}{ll}
\hline Treatment & $\begin{array}{c}\text { Increase of } \\
\text { height }(\mathrm{cm})\end{array}$ \\
\hline SNaKd $330 \mathrm{~W}$ & \\
peat & $18.7 \mathrm{a}$ \\
peat-perlite $3+1$ & $15.2 \mathrm{ab}$ \\
peat-perlite $2+2$ & $15.3 \mathrm{ab}$ \\
$\mathrm{HgLX} 400 \mathrm{~W}$ & \\
peat & $13.5 \mathrm{ab}$ \\
peat-perlite $3+1$ & $16.0 \mathrm{ab}$ \\
peat-perlite $2+2$ & $14.2 \mathrm{ab}$ \\
HPI/T $375 \mathrm{~W}$ & \\
peat & $14.0 \mathrm{ab}$ \\
peat-perlite $3+1$ & $15.9 \mathrm{ab}$ \\
peat-perlite $2+2$ & $13.8 \mathrm{ab}$ \\
Incandescent $100 \mathrm{~W}$ & \\
peat & $13.3 \mathrm{ab}$ \\
peat-perlite $3+1$ & $12.1 \mathrm{~b}$ \\
peat-perlite $2+2$ & $10.9 \mathrm{~b}$ \\
Mean & 14.4 \\
\hline
\end{tabular}

Means marked with the same letter in one column (all treatments) do not differ at the $5 \%$ rate of error. noted in the increase of height in the cultivar 'Afrodite' (Table 7). The diameter of the root collar was not greatly affected by the different lighting and substrates, but in many cultivars it was greatest in plants lighted with HPI/T lamps and smallest in plants grown under incandescent lamps (Table 9). The effect of a treatment on the proportion of saleable plants depended closely on the cultivar and showed great variation (Table 10).

\section{Discussion}

The results obtained at the end of lighting show that plants lighted with $\mathrm{SNaKd}$ lamps were generally higher and broader than those in the other lighting groups. Light from $\mathrm{SNaKd}$ lamps contains a greater proportion of red wavelengths than light from HPI/T or $\mathrm{HgLX}$, which is reported to cause elongation growth (ANON. 1973, SANDAHL 1979). HageN (1978) observed elongation in response to Philips SON/T, which is also a high-pressure sodium lamp. MoE (1985), however, obtained different results when growing Elatior begonias in a growth chamber. He reports that $\mathrm{HPI} / \mathrm{T}$ caused more elongation than SON/T. The elongation and poor increase of width in plants grown under incandescent lamps were natural consequences of the low light intensities. 
Table 8. Effect of lighting and substrate on the final increase of width $(\mathrm{cm})$ in Elatior begonias.

\begin{tabular}{|c|c|c|c|c|c|c|c|c|c|}
\hline \multirow[b]{2}{*}{ Treatment } & \multicolumn{8}{|c|}{ Cultivar } & \multirow[b]{2}{*}{$\begin{array}{c}\text { Treatment } \\
\text { mean }\end{array}$} \\
\hline & 'Afrodite' & $\begin{array}{l}\text { 'Afrodite } \\
\text { Special' }\end{array}$ & $\begin{array}{l}\text { 'Afrodite } \\
\text { Rosa' }\end{array}$ & 'Mandela' & 'Barbara' & 'Connie' & 'Marja' & 'Sirene' & \\
\hline \multicolumn{10}{|l|}{ Lighting: } \\
\hline SNaKd $330 \mathrm{~W}$ & $21.4 \mathrm{a}$ & $25.0 \mathrm{a}$ & $20.6 \mathrm{a}$ & $15.8 \mathrm{a}$ & $22.2 \mathrm{a}$ & $12.5 \mathrm{a}$ & $14.5 \mathrm{a}$ & $10.3 \mathrm{a}$ & 17.8 \\
\hline $\mathrm{HgLX} 400 \mathrm{~W}$ & $18.2 \mathrm{~b}$ & $25.3 \mathrm{a}$ & $17.3 \mathrm{ab}$ & $15.9 \mathrm{a}$ & $22.0 \mathrm{a}$ & $11.6 \mathrm{ab}$ & $12.5 b$ & $10.7 \mathrm{a}$ & 16.7 \\
\hline $\mathrm{HPI} / \mathrm{T} 375 \mathrm{~W}$ & $18.0 \mathrm{~b}$ & $26.2 \mathrm{a}$ & $18.9 \mathrm{a}$ & $15.0 \mathrm{a}$ & $21.3 \mathrm{a}$ & $10.8 \mathrm{ab}$ & $11.3 \mathrm{~b}$ & $9.9 \mathrm{ab}$ & 16.4 \\
\hline incandescent $100 \mathrm{~W}$ & $16.3 b$ & $24.0 \mathrm{a}$ & $15.5 b$ & $14.4 \mathrm{a}$ & $19.3 \mathrm{a}$ & $9.9 \mathrm{~b}$ & $10.9 b$ & $8.0 \mathrm{~b}$ & 14.8 \\
\hline \multicolumn{10}{|l|}{ Substrate: } \\
\hline peat & $18.8 \mathrm{a}$ & $24.7 \mathrm{a}$ & $18.3 \mathrm{a}$ & $16.4 \mathrm{a}$ & $21.3 \mathrm{a}$ & $11.6 \mathrm{ab}$ & $12.5 \mathrm{a}$ & $10.0 \mathrm{a}$ & 16.7 \\
\hline peat-perlite $3+1$ & $18.1 \mathrm{a}$ & $24.2 \mathrm{a}$ & $18.1 \mathrm{a}$ & $14.7 \mathrm{a}$ & $20.9 \mathrm{a}$ & $11.8 \mathrm{a}$ & $12.2 \mathrm{a}$ & $9.7 \mathrm{a}$ & 16.2 \\
\hline peat-perlite $2+2$ & $18.5 \mathrm{a}$ & $27.0 \mathrm{a}$ & $17.9 \mathrm{a}$ & $14.8 \mathrm{a}$ & $21.5 \mathrm{a}$ & $10.2 \mathrm{~b}$ & $12.2 \mathrm{a}$ & $9.4 \mathrm{a}$ & 16.4 \\
\hline Cultivar mean & 18.5 & 25.2 & 18.1 & 15.3 & 21.2 & 11.2 & 12.3 & 9.7 & \\
\hline
\end{tabular}

Means marked with the same letter in one column do not differ at the $5 \%$ rate of error.

Table 9. Effect of lighting and substrate on the diameter of the root collar $(\mathrm{mm})$ in Elatior begonias.

\begin{tabular}{|c|c|c|c|c|c|c|c|c|c|}
\hline \multirow[b]{2}{*}{ Treatment } & \multicolumn{8}{|c|}{ Cultivar } & \multirow[b]{2}{*}{$\begin{array}{c}\text { Treatment } \\
\text { mean }\end{array}$} \\
\hline & 'Afrodite' & $\begin{array}{l}\text { 'Afrodite } \\
\text { Special' }\end{array}$ & $\begin{array}{l}\text { 'Afrodite } \\
\text { Rosa' }\end{array}$ & 'Mandela' & 'Barbara' & 'Connie' & 'Marja' & 'Sirene' & \\
\hline \multicolumn{10}{|l|}{ Lighting: } \\
\hline SNaKd $330 \mathrm{~W}$ & $5.6 \mathrm{a}$ & $6.0 \mathrm{a}$ & $5.2 \mathrm{a}$ & $7.2 \mathrm{a}$ & $6.0 \mathrm{a}$ & $8.0 \mathrm{a}$ & $7.6 \mathrm{a}$ & $6.5 \mathrm{a}$ & 6.5 \\
\hline $\mathrm{HgLX} 400 \mathrm{~W}$ & $5.5 \mathrm{a}$ & $5.8 \mathrm{a}$ & $5.2 \mathrm{a}$ & $6.2 \mathrm{bc}$ & $6.4 \mathrm{a}$ & $8.3 \mathrm{a}$ & $7.8 \mathrm{a}$ & $6.7 \mathrm{a}$ & 6.5 \\
\hline $\mathrm{HPI} / \mathrm{T} 375 \mathrm{~W}$ & $5.9 \mathrm{a}$ & $6.2 a$ & $5.4 \mathrm{a}$ & $6.9 \mathrm{ab}$ & $6.6 a$ & $7.7 \mathrm{a}$ & $7.9 \mathrm{a}$ & $6.9 \mathrm{a}$ & 6.7 \\
\hline incandescent $100 \mathrm{~W}$ & $5.1 \mathrm{a}$ & $5.4 \mathrm{a}$ & $5.2 \mathrm{a}$ & $5.9 \mathrm{c}$ & $5.7 \mathrm{a}$ & $7.5 \mathrm{a}$ & $7.0 \mathrm{a}$ & $6.2 \mathrm{a}$ & 6.0 \\
\hline \multicolumn{10}{|l|}{ Substrate: } \\
\hline peat & $5.7 \mathrm{a}$ & $5.7 \mathrm{a}$ & $5.3 \mathrm{a}$ & $6.8 \mathrm{a}$ & $6.4 \mathrm{a}$ & $7.9 \mathrm{a}$ & $7.0 \mathrm{a}$ & $6.3 \mathrm{a}$ & 6.4 \\
\hline peat-perlite $3+1$ & $5.6 \mathrm{a}$ & $6.1 \mathrm{a}$ & $5.3 \mathrm{a}$ & $6.9 \mathrm{a}$ & $6.0 \mathrm{a}$ & $8.0 \mathrm{a}$ & $8.0 \mathrm{~b}$ & $7.0 \mathrm{a}$ & 6.6 \\
\hline peat-perlite $2+2$ & $5.3 \mathrm{a}$ & $5.7 \mathrm{a}$ & $5.0 \mathrm{a}$ & $6.0 \mathrm{~b}$ & $6.1 \mathrm{a}$ & $7.8 \mathrm{a}$ & $7.7 \mathrm{ab}$ & $6.4 \mathrm{a}$ & 6.2 \\
\hline Cultivar mean & 5.5 & 5.8 & 5.2 & 6.6 & 6.2 & 7.9 & 7.6 & 6.6 & \\
\hline
\end{tabular}

Means marked with the same letter in one column do not differ at the $5 \%$ rate of error.

Table 10. Effect of lighting and substrate on the proportion $(\%)$ of saleable plants in Elatior begonias.

\begin{tabular}{|c|c|c|c|c|c|c|c|c|c|}
\hline \multirow[b]{2}{*}{ Treatment } & \multicolumn{8}{|c|}{ Cultivar } & \multirow[b]{2}{*}{$\begin{array}{c}\text { Treatment } \\
\text { mean }\end{array}$} \\
\hline & 'Afrodite' & $\begin{array}{l}\text { 'Afrodite } \\
\text { Special' }\end{array}$ & $\begin{array}{l}\text { 'Afrodite } \\
\text { Rosa' }\end{array}$ & 'Mandela' & 'Barbara' & 'Connie' & 'Marja' & 'Sirene' & \\
\hline \multicolumn{10}{|l|}{ Lighting: } \\
\hline SNaKd $330 \mathrm{~W}$ & 28 & 0 & 44 & 67 & 50 & 50 & 22 & 88 & 43 \\
\hline $\mathrm{HgLX} 400 \mathrm{~W}$ & 33 & 0 & 28 & 44 & 53 & 33 & 22 & 78 & 36 \\
\hline $\mathrm{HPI} / \mathrm{T} 375 \mathrm{~W}$ & 81 & 0 & 33 & 44 & 19 & 44 & 11 & 67 & 37 \\
\hline incandescent $100 \mathrm{~W}$ & 50 & 6 & 50 & 39 & 47 & 33 & 39 & 44 & 38 \\
\hline \multicolumn{10}{|l|}{ Substrate: } \\
\hline peat & 62 & 4 & 38 & 46 & 57 & 29 & 29 & 67 & 41 \\
\hline peat-perlite $3+1$ & 54 & 0 & 42 & 54 & 38 & 46 & 12 & 74 & 40 \\
\hline peat-perlite $2+2$ & 26 & 0 & 38 & 46 & 32 & 46 & 29 & 67 & 35 \\
\hline Cultivar mean & 47 & 1 & 39 & 49 & 42 & 40 & 24 & 69 & \\
\hline
\end{tabular}


When observed 4 weeks after potting, plants potted in peat or peat-perlite $3+1$ had generally grown better than plants potted in peat-perlite $2+2$. This was to be expected, because the more perlite there was in the substrate mixture, the lower was the amount of available nutrients.

The earliest flowering and arrival at the sale stage were obtained with SNaKd and $\mathrm{HgLX}$ lamps. HAGEN (1978) also reports that highpressure sodium lamps (SON/T) accelerate flowering, but MoE (1985) did not discover any notable differences between SON/T and HPI/T in this respect. The effect of the substrate on anthesis and arrival at the sale stage was most pronounced in the cultivar 'Afrodite Special': anthesis began 2 weeks later in peat-perlite $2+2$ than in other substrates. In most cultivars, however, the differences were not significant.

At the sale stage, the highest and the broadest plants were in most cases those grown under SNaKd lamps, which coincides with the results at the end of lighting. Surprisingly, the differences between the substrate groups did not increase, rather the opposite. It seems that regular fertilization, begun 3 weeks after potting, evened out the differences. The diameter of the root collar was not greatly affected by the different lighting or substrates. In plants of the vigorously growing cultivars, i.e. the 'Afrodite' group and 'Mandela', the root collars tended to be too thin to support the heavy crown and as a rule they needed support. The thinness of the root collar is a special problem in micropropagated Elatior begonias and a cause of dissatisfaction among the commercial growers.

The proportion of saleable plants varied greatly with the treatment and the cultivar. Hagen (1978) reports that different lamp types do not cause significant differences in the final appearance or quality of Elatior begonias. From the standpoint of commercial production, the total proportion of saleable plants in this experiment was rather low. The main factors reducing the saleability were long internodes, and leaf and flower stalks ('Afro- dite'), strong vegetative growth and weak flowering ('Afrodite Special', 'Afrodite Rosa' and 'Mandela'), an oblique growth habit, which means that the plants bend to one side and form a front and a back ('Barbara', 'Connie' and 'Marja'), and short flower stalks ('Sirene'). The long internodes, and leaf and flower stalks of the cultivar 'Afrodite' could probably be overcome by using an appropriate growth regulator and lower temperatures. The short flower stalks of 'Sirene' could be overcome by using higher temperatures. The choice of temperature can be a problem if cultivars of different growth vigour are grown in the same space.

The abundant vegetative growth and the oblique growth represent more difficult problems. Strong vegetative growth and branching caused a bushlike appearance and were connected with weak, delayed flowering. This was observed especially in the cultivar 'Afrodite Special'; $15 \%$ of these plants failed to reach the sale stage before the experiment was terminated. It was often noticed that flowers and flower buds were formed only on the oldest branch and that the new, vigorous branches were completely vegetative. Oblique growth was not restricted to the vigorously growing cultivars with a heavy crown, in which it could be overcome by supporting the crown with a couple of sticks, but was also a problem in the cultivars 'Connie' and 'Marja', the plants of which were otherwise quite sturdy with thicker root collars. Oblique growth was not observed to be caused by the direction of the incoming daylight and the reason for this growth habit is not clear.

Vigorous growth and delayed flowering are often reported to be the result of abundant fertilization. It is possible that, due to their healthiness, micropropagated Elatior begonias do not require as much fertilizer as the traditionally propagated plants. There are also differences between cultivars in this respect. Another possible cause of strong vegetative growth could be the after-effect of hormones or other substances used in the tissue culture media. Very little research has been devoted 
to the after-effects of tissue culture media, possibly because micropropagation is a relatively new method. Further studies are needed to elucidate the reasons for the unwanted growth habits of certain micropropagated Ela-

\section{References}

ANON. 1973. Lighting in Greenhouses. Growelectric handbook n:o 2. 98 p. London.

- 1987. Puutarha-alan vuosikirja 1986. 96 p. Joensuu.

Bıgot, C. 1981. Multiplication végétative in vitro de Begonia $\times$ hiemalis ('Rieger' et 'Schwabenland'). II. Conformité des plantes élevées en serre. Agronomie 1: $441-447$.

HAGEN, P. 1978. Sammenligning av lysstoffrør, høytrykkvikksølvhalogen- og høytrykknatriumdamplamper som tilleggslys til Hiemalisbegonia i vinterhalvåret. Norges Landbrukshøgsk., Inst. for blomsterdyrking og veksthusforsøk, Meld. 213.

Hilding, A. \& Welander, T. 1976. Effects of some Factors on Propagation of Begonia $\times$ hiemalis in vitro. Swedish J. agric. Res. 6: 191-199.

-1982 . Produktion av Begonia $\times$ elatior. Sveriges tior begonia cultivars.

Acknowledgements. The author would like to thank the horticultural foundation of Nikolai and Ljudmila Borisoff for financial support, and OY Hortus AB for cooperation.
Lantbruksuniv., Konsulentavd. rapp., Trădgård 220. Alnarp.

MOE, R. 1985. Licht an für Jungpflanzen von ElatiorBegonien. Gb + Gw 85: 205-206.

SANDAHL, B. 1979. Belysning av växter. Sveriges Lantbruksuniv., Konsulentavd. rapp., Trädgård 176. Alnarp.

Welander, T. 1978. In vitro Propagation of Clones from Different Cultivars of Begonia $\times$ hiemalis. Swedish $\mathbf{J}$. agric. Res. 8: 181-186.

- 1987. Odlingsförsök med mikroförökad Begonia $\times$ elatior hybr. 'Elfe'. Sveriges Lantbruksuniv., Konsulentavd. rapp., Trädgård 318. Alnarp.

Ms received October 5, 1987

\section{SELOSTUS}

\section{Mikrolisätyn pauliinabegonian (Begonia $\times$ hiemalis) kasvu ja kehitys in vivo. I. Valotuksen ja kasvualustan vaikutus.}

\section{Päivi Roivainen \\ Helsingin yliopisto, puutarhatieteen laitos 00710 Helsinki}

Mikrolisãyksellă voidaan saada aikaan terveitä pauliinabegonian taimia. Niiden jatkokasvatuksessa on kuitenkin ilmennyt ongelmia. Taimet rehevooityvăt liikaa, niiden juurenniska jảă ohueksi ja kukinta viivästyy.

Viljelytekniikan vaikutuksen selvittämiseksi järjestettiin kevaaalllă 1987 valotus- ja kasvualustakoe seuraavilla mikrolisătyillä pauliinabegonialajikkeilla: 'Afrodite', 'Afrodite Special', 'Afrodite Rosa', 'Mandela', 'Barbara', 'Connie', 'Marja' ja 'Sirene'. Kokeessa oli mukana neljă eri lampputyyppiä: suurpainenatriumlamput (Airam SNaKd $330 \mathrm{~W}$ ), suurpaine-elohopealamput (Airam $\mathrm{HgLX}$ $400 \mathrm{~W}$ ), monimetallilamput (Philips HPI/T 375 W) sekả kontrollina hehkulamput $100 \mathrm{~W}$. Kokeessa mukana olleet kasvualustaseokset olivat B2-turve, B2-turve ( 3 osaa) plus perliitti ( 1 osa) sekä B2-turve ( 2 osaa) plus perliitti ( 2 osaa).

$\mathrm{SNaKd}$ - tai $\mathrm{HgLX}$-lampuilla valotetut taimet olivat korkeimpia, leveimpiă sekä aloittivat kukinnan ja saavuttivat myyntikuntoisuuden aikaisimmin. Kontrolliryhmän taimet olivat pienimpiả sekä kukkivat ja tulivat myyntikuntoisiksi viimeisină. Kasvualustaseosten vaikutus kukinnan alkamisen ja myyntikuntoisuuden saavuttamisen ajankohtaan näkyi selvästi ainoastaan 'Afrodite Special' -lajikkeella, jonka turpeen ja perliitin $2+2$-seoksessa 
kasvaneet taimet kukkivat 2 viikkoa myöhemmin muilla alustoilla kasvatettuihin taimiin verrattuna. Taimien korkeuteen ja leveyteen eri kasvualustaseoksilla ei ollut kovinkaan suurta vaikutusta. Mikäăn lampputyypeistä tai kasvualustaseoksista ei muihin verrattuna lisännyt selvăsti kukkien ja nuppujen lukumäărăă tai juurenniskan lăpi- mittaa. Myyntikelpoisten kasvien osuus vaihteli suuresti lajikkeesta, valotuksesta ja kasvualustasta riippuen. Useimmilla lajikkeilla myyntikelpoisia kasveja oli alle puolet kokonaismäärästă. Myyntikelpoisuutta vähensivät ennenkaikkea liiallinen rehevyys, heikko kukinta ja vino kasvutapa. 